\title{
Mechanism Design of Surmounting Obstacles Walking Machine
}

\author{
Min Zeng \\ Nanchang Institute of Science \& Technology
}

\begin{abstract}
Keywords: Mechanical engineering; Walking surmounting obstacle system; Mechanical design; Deicing robot; Control system
\end{abstract}

\begin{abstract}
Ice covering high voltage transmission lines caused by the frozen snow disaster brought great harm to our power system and great loss to people's production and life, so the study of high-voltage transmission line deicing robot is of great practical significance. This paper puts forward a new type of high voltage line deicing robot structure, mainly for its walking surmounting obstacle system, designs and makes the prototype thorough the actual environment of high voltage line. The paper also establishes relevant mathematics models for online walking surmounting obstacles, and verifies the feasibility of this design through three steps of theoretical analysis, simulation modeling and practical experiments.
\end{abstract}

\section{Introduction}

As is known to all, China is a country needing more electric power, and the smooth development of all industries is inseparable from the power system. At the same time, our country is also one of the countries with severe conditions of transmission lines ice cover. Freezing rain and snow and other natural disasters make the transmission lines covered by ice, causing transmission line break and pole down, resulting in power cut in the around area and more wide areas, thus making national economy and people's life suffered a heavy blow.

Generally speaking, there are three kinds of deicing methods for high-voltage power lines, namely artificial deicing, mechanical d-icing and DC heat melting ice. These methods have too high cost that is not suitable for long distance deicing; for on-line operation, not only inefficient, but the risk is extremely high. Pulley scaling method is put forward by Canada. It is a mechanical de-icing method, and this online ice removal method is done by pulley through the ground operators pulling it. The biggest advantage is simple operation and low cost, but its defect is low efficiency and cannot be used in complex terrain and high altitude area. Most research of deicing robots that can surmount obstacles is still in a laboratory experimental study phase. Because there are a variety of practical problems, no molding products emerge. So, now we need to research automatic deicing robots on transmission line, which cannot only operate safely and steadily on the transmission line, but also can automatically deice and surmount the obstacle. It has very good practical value.

\section{Mechanism Design of Walking Surmounting Obstacles}

Robot mechanical structure design is the base of the whole system design. For the high voltage line deicing robot, in addition to the stable, compact structure, it should have the ability surmounting the obstacles. Most of the mobile mechanism can't surmount the obstacle, and is generally completed relying on other auxiliary mechanisms. Integrating the references, common auxiliary mechanism has the following three forms:

1) Humanoid arm climbing mechanism, its working process is to hang on both ends of obstacles with its auxiliary body, and the robot glides along the arm rail, so as to surmount the obstacles. Its characteristic is the arm has great rotary scale space that the robot can span a longer size obstacles, etc, but the main structure is complex and has high weight and energy consumption, so it's hard to climb the slope above $30^{\circ}$, and the application scope is small.

2) Multiple fission mechanism, its "head decision-making, tail body follow" control strategy makes sure every single section pass the obstacles with certain order, so it has strong ability of surmounting obstacles, but the shortage is that it can only surmount the obstacles of small size and 
small span. The multi-joints lead it to have more degrees of freedom, and complex mechanism leads to the complexity of control system, also it moves slowly with low efficiency, therefore it cannot be widely used.

3) Composite structure with wheel and arm, which is used more by the domestic inspection robot, there are two such mechanisms: three arms and two arms. Comparing with the former two types, this structure is more stable and compact with less weight; comparing with the multiple fission mechanism, it is simple, reliable, and the control strategy is more concise and has more efficient movement.

\section{Mechanism Structure}

Combining with the basic condition of the high voltage line and the type and size of obstacles, and integrating the load bearing characteristics of high voltage lines, we puts forward certain requirements on robot structure design. The distance to the ground is generally $30 \sim 50 / \mathrm{W}$, where the wind is very strong and the span of pylons is larger, so once the external environment (such as wind) sudden change is bound to cause vibration of the transmission line. To this end, we put forward the related design technical indexes and requirements:

(1) The total weight cannot exceed the required weight in the operation to save the energy;

(2) The robot should be as small as possible, making it be compact to enhance the stability;

(3) In addition to meet the structure design requirements, the space for installing control box and related sensors should be enough and the electrical interface should be reserved;

(4) Ensure certain walking speed, which can reach $0.4 \mathrm{~km} / \mathrm{h}$ that the work efficiency can be improved greatly;

(5) Ensure certain climbing gradient and it should have the gradeability of $25^{\circ}$ (aim at $30^{\circ}$ in actual design )

(6) Have the ability to hold the transmission line; when meeting the hostile environment, it can stop and hold the high voltage lines tightly to improve its safety;

(7) It should have obstacle climbing ability, which makes sure it can surmount the quakeproof and suspension clamp and other obstacles safely and stably.

\section{Design of the Core Components}

Clamping Jaw Design. Clamping jaw must be reliable and stable to clamp transmission lines without the phenomenon of slippage. Under the condition of bad external environment, it can clamp the lines timely, and the body is not overturned. Therefore, the design of clamping jaw should meet the requirements: in the process of robot surmounting obstacles, adequate clamping force should be ensured, and relative sliding between the head and wire cannot occur; second, make sure the wire doesn't fall off from the jaw in order to prevent friction of the bottom and wire when the plate rotating in surmounting the obstacles.

After calculation, the equation is concluded as following:

$$
\begin{gathered}
M=F * \frac{L_{1} L_{4}}{L_{3}} * \frac{\sin a}{\sin b} \\
M=M_{\text {电机 }} * \mathrm{n}
\end{gathered}
$$

After subtracting one by another:

In the equation:

$$
M_{\text {电机 }}=\mathrm{F} * \frac{L_{1} L_{4}}{n L_{3}} * \frac{\sin a}{\sin b}
$$

$\mathrm{F}$ - - the clamping force of the jaw;

$L_{1}, L_{3}, L_{4}, a, b$ - the length of each connecting rod and the included angle among各连杆 them;

$\mathrm{n}-$ - reduction ratio of gears. 
Walking System. Walking mechanism is composed by ball screw nut mechanism, DC servo motor, belt wheel, etc. Implementation process: DC servo motor drives belt wheel, and then make the screw rotate, the nut moving along the lead screw rail back and forth, combining three clamping jaws holding and loosing lines to realize the forward motion of the machine.

Because the whole machine undertakes the biggest load in the upslope stage, namely the middle clamping jam holds the transmission lines with front and back two clamping jams open, the wheel system of surmounting obstacles plate is driven by the bottom motor to go forward. Now, the walking motor on the bottom need to overcome the resistances which include the component forces along bottom plate, the friction between the wheel and transmission line, and the friction of screw nut mechanism. Firstly, model simplification; the forces are analyzed as following:

$$
\begin{aligned}
& \mathrm{F}-\mathrm{f}_{1}-\mathrm{f}_{2}-m *(g \sin \alpha+a)=0 \\
& N_{1}+N_{2}-m g \cos \alpha=0 \\
& f_{1} \leq \mu N_{1} \\
& f_{2} \leq \mu N_{2}
\end{aligned}
$$

In the formula,

$f_{1}, f_{2}$ - force of rolling friction between the obstacle crossing plate and the transmission line;

$N_{1}, N_{2}$ - - reverse force of the positive pressure on transmission line by the front and前后越 back wheels ;

$\mu-$ coefficient of rolling friction between the back and front obstacle crossing plate and transmission line.

The following formula can be got from the formulas above:

$$
F=m *(g \sin \alpha+a)+\mu m g \cos \alpha
$$

\section{Conclusion}

This paper adopts the obstacle-surmounting plate and clamping jaw mechanism to realize the robot walking and surmounting obstacle function. Three clamping jaws open and close regularly to achieve forward movement; the obstacle-surmounting plate can not only ensure the stationarity of robot in running and at least two points on the high voltage line. In normal operation, the obstacle-surmounting plate reduces the friction with transmission lines, so as to reduce the driving force of motor; the paper establishes relevant mathematical models, analyzing the conditions for uphill, downhill and obstacle-surmounting. During normal operation, the middle clamping jaw slightly lower than the front and rear clamping jaw is more advantageous to the robot running stably.

\section{References}

[1] Pyramid A. Kecskemet College - Faculty of Mechanical Engineering and Automation [J]. Science \& Engineering of Composite Materials, 2011, 4(6):711-721.

[2] $\mathrm{Xu} \mathrm{L}, \mathrm{Pu} \mathrm{Q}$, University N P. The development direction of mechanical engineering and automation [J]. Electronic Test, 2014.

[3] Dong H, University N P. Mechanical Design, Manufacturing and Automation direction-Study mechanical engineering and automation development direction [J]. Electronic Test, 2014.

[4] Gao X, Deng Z. Practice and Research of Dominant Specialty of Mechanical Engineering and Automation [J]. Value Engineering, 2012. 
[5] Wang S Z, Liang W U, Hong-Bo G U, et al. Reforming on the Educational System of Engineering Practice for Mechanical Engineering and Automation Speciality [J]. Research \& Exploration in Laboratory, 2005.

[6] Wang Q G. Construction and Practice of Innovation Talent Cultivation System of Mechanical Engineering and Automation [J]. Meitan Higher Education, 2009.

[7] Qin C J, Huang Z Y. Reform of Production Practice in Mechanical Engineering and Automation [J]. Research \& Exploration in Laboratory, 2006.

[8] Voskoboĭnikov, B. S. (Boris Solomonovich). English-Russian dictionary of mechanical engineering and industrial automation: about 100000 terms [M]. "Pycco", 2001.

[9] Mohanty H K, Venkateswarlu D, Mahapatra M M, et al. Modeling the Effects of Tool Probe Geometries and Process Parameters on Friction Stirred Aluminium Welds [J]. Journal of Mechanical Engineering \& Automation, 2012, 2(4):74-79.

[10]Ji K, Kim W. Optimal Bandwidth Allocation and QoS-adaptive Control Co-design for Networked Control Systems [J]. International Journal of Control Automation and System Vol.6 No.4, 2007, 6(4):831-839.

[11]Herman V D A. Development and evaluation of advanced measurement methods for experimental modal analysis [J]. Production Engineering Machine Design \& Automation, 1987. 